\title{
THE TRANSITION-RADIATION AS A LIGHT-SOURCE IN THE VUV
}

\author{
W. BÖHM and D. LABS \\ Landessternwarte, Heidelherg-Königstuhl, Germany
}

Using the principle of the 'transition-radiation', it is possible to construct a new lightsource for the VUV. This principle states that an electromagnetic radiation is emitted, when an electric charge penetrates the surface of a metallic target. The essential advantages of such a source are that it emits a continuous spectrum in the whole region from 1000 to $3000 \AA$ and that this radiation is emitted directly in the vacuum.

An electron gun fires electrons with an energy of about $10-15 \mathrm{keV}$ onto a water cooled aluminium-target. The presence of the radiation (after passing through a grating monochromator) is indicated by a photomultiplier. One gets a signal down to $1050 \AA$ (the cutoff of the multiplier window).

An absolute calibration in the region 1700-2700 $\AA$ was made by comparing the transition-radiation with that of a calibrated deuterium-lamp. (The full paper will be published in the Applied Optics (issue September 1971).)

\section{DISCUSSION}

$R$. J. Van Duinen: Seeing the amount of power dissipated in the target is there any thermal effect on your calibration? (like thermal radiation and change of optical parameters).

$D$. Labs: The target is water cooled, so that we have no strong thermal radiation. In addition, the multiplier used was solar-blind.

B. Bates: Are there any other materials which might be useful for the electron beam target?

D. Labs: We work also with tungsten and platinum as target materials; but aluminium emits more intensity than the other ones. 